\title{
RAIRO
}

ANALYSE NUMÉRIQUE

\section{MANFRED DOBROWOLSKI}

\section{Convergence of finite element approximation to} quasilinear initial boundary value problems

RAIRO - Analyse numérique, tome 12, no 3 (1978), p. 247-266.

<http://www.numdam.org/item?id=M2AN_1978_12_3_247_0>

(C) AFCET, 1978, tous droits réservés.

L'accès aux archives de la revue «RAIRO - Analyse numérique » implique l'accord avec les conditions générales d'utilisation (http://www.numdam.org/ legal.php). Toute utilisation commerciale ou impression systématique est constitutive d'une infraction pénale. Toute copie ou impression de ce fichier doit contenir la présente mention de copyright.

\section{Numdam}




\title{
$L^{\infty}$-CONVERGENCE OF FINITE ELEMENT APPROXIMATION TO OUASILINEAR INITIAL BOUNDARY VALUE PROBLEMS (*)
}

\author{
by Manfred DobrowolsKI $\left({ }^{1}\right)$
}

Communiqué par P -A RaviarT

Abstract - Almost opttmal $L^{\infty}$-convergence of fintte element approximation to nonlinear parabolic differential equations is proved by a weighted norm technique known from the elliptic case

\section{INTRODUCTION AND STATEMENT OF THE THEOREM}

In this paper, we study the finite element approximation of nonlinear parabolic problems. Our aim is to obtain optimal uniform convergence for the discrete solution. As a model problem we consider the parabolic initial boundary value problem:

$$
\left\{\begin{array}{c}
u_{t}-\sum_{\imath=1}^{n} \partial_{\imath} F_{\imath}(\nabla u)=f(x, t) \quad \text { in } \Omega \times[0, T], \\
u(x, 0)=\psi(x) \quad \text { in } \Omega, \quad u(x, t)=0 \quad \text { on } \partial \Omega \times[0, T] .
\end{array}\right.
$$

In the above, $\Omega$ is a bounded domain in Euclidian $\mathbf{R}^{n}$ with sufficiently smooth boundary $\partial \Omega$.

Error estimates for quasilinear parabolic equations have been proved in various papers. J. Douglas Jr. and T. Dupont [6] have established optimal $H^{1-}$ error estimates and V. Thomee and L. Wahlbin [15] have shown that the nonlinearity must not fulfil a global Lipschitz condition. Optimal $L^{2}$-error estimates, i. e. $\left\|e_{h}\right\|_{L^{2}}=O\left(h^{m}\right)$, have been obtained by Wheeler [16]. However the nonlinearity treated by Wheeler involves only $u$ and not $\nabla u$. Optimal pointwise estimates have been established by Bramble, Schatz, Thomee and Wahlbin [2] in the linear case $\left(u_{t}=A u\right)$ and by Dobrowolski [5] in the quasilinear case treated by Wheeler for $n=2$, in the general linear case for $n \geqq 2$.

(*) Reçu septembre 1977

(1) Institut fur Angewandte Mathematık und Informatık, Unıversitàt Bonn, Bonn, République Fédérale d'Allemagne 
$H^{j, p}(\Omega), j \in \mathbf{N}, 1 \leqq p<\infty$, denotes the completion of $C^{\infty}(\Omega)$ with respect to the norm:

$$
\|w\|_{j, p}=\sum_{i=0}^{j}\left(\int_{\Omega}\left|\nabla^{i} w\right|^{p} d x\right)^{1 / p}
$$

If this norm is taken in a domain $T \subset \mathbf{R}^{n}$ we shall write $\|\cdot\|_{j, p, T}$. In the case $j=0$ and $p=2$ we will write $\|\cdot\|_{T}$. Furthermore we use $H_{0}^{1}(\Omega)$, the closure of $C_{0}^{\infty}(\Omega)$ in $H^{1}(\Omega)$, and $H^{j, \infty}(\Omega)$, the space of functions with bounded generalized derivatives. Let $X$ be a normed linear space consisting of a set of functions defined on $\Omega$. If $w$ is a function defined on $[0, T] \times \Omega$ we say $w \in L^{p}(X), 1 \leqq p<\infty$, if the norm:

is finite where:

$$
\|w\|_{L^{p}(X)}=\left(\int_{0}^{T}|F(t)|^{p} d t\right)^{1 / p}
$$

$$
F(t)=\|w(t)\|_{X},
$$

with the usual modification for $p=\infty$.

For abbreviation, we write $\|\cdot\|_{\infty}$ instead of $\|\cdot\|_{L^{\infty}\left(L^{\infty}\right)}$ and use a summation convention.

With the above notation we state our assumptions concerning problem $(P)$ :

$$
F_{i} \in C^{3}\left(\mathbf{R}^{n}\right), i=1, \ldots, n, f \in C^{2}\left(\Omega \times[0, T], \psi \in H^{m, \infty}(\Omega) .\right.
$$

(A2) Ellipticity (not necessarily uniform): for $p, \xi \in \mathbf{R}^{n}, \xi \neq 0$, and $|p| \leqq K$ there is a constant $C(K)$ with $F_{i, k}(p) \xi_{i} \xi_{k} \geqq C(K)|\xi|^{2}$ where $\left(F_{i k}\right)$ denotes the matrix of the first derivatives of $F_{i}$.

$$
\sum_{i=1}^{n} F_{i}(0)=0
$$

(A4) There is a unique solution $u$ of the problem $(P)$ and $u(., t)$, $u_{t}(., t) \in H^{m, \infty}(\Omega), t \in[0, T], u_{t t} \in L^{2}\left(H^{m, \infty}(\Omega)\right)$.

(A5) If the data of problem $(P)$ are replaced by $r f(x, t)$ and $r \psi(x), r \in[0,1]$, the solution $u^{r}$ sufficies assumption (A4) and is bounded in the norms of (A4) uniformly in $r$. In particular, we have $\left\|\nabla u^{r}\right\|_{\infty},\left\|\nabla u_{t}^{r}\right\|_{\infty} \leqq \gamma$. Moreover $u^{r}$ continuously depends on $r$.

Remark: The $m$ in the assumptions refers to the order of the spline space, which will be described below.(A3) is superfluous, but it allows us to give a more elegant presentation of the proof.

We shall assume that the spline space $S_{h}, 0<h \leqq h_{0}$, of order $m$ satisfies the following conditions:

(S1) $S_{h}$ is a finite dimensional subspace of $H_{0}^{1}(\Omega)$. 
(S2) There is a partition $\tau_{h}$ of $\Omega$ into piecewise smooth subdomains such that the usual regularity condition is fulfilled: each $T \in \tau_{h}$ is contained in a ball of radius $h$ and contains a ball of radius $m h$ where the constant $m>0$ does not depend on $h$.

For all subdomains $T \in \tau_{h}$ and for all $z_{h} \in S_{h}$ we have $z_{h} \in H^{m}(T)$.

(S3) The following approximation and inverse properties hold:

(i) to each $z \in H^{m, p} \cap H_{0}^{1}$, there is a function $z_{h} \in S_{h}$ such that on each $T \in \tau_{h}$ :

$$
\left\|z-z_{h}\right\|_{j, p, T} \leqq \mathrm{ch}^{m-j}\|z\|_{m, p, T^{\prime}}, \quad 0 \leqq j \leqq m, \quad 1 \leqq p \leqq \infty,
$$

where $T^{\prime}:=T \cup\{$ neighbours of $T\}$;

(ii) for $z_{h} \in S_{h}$ and $T \in \tau_{h}$ we have:

$$
\begin{gathered}
\left\|z_{h}\right\|_{k, p, T} \leqq \mathrm{ch}^{-k}\left\|z_{h}\right\|_{p, T}, \quad 0<k<m, \quad 1 \leqq p \leqq \infty, \\
\left\|z_{h}\right\|_{j, \infty, T} \leqq \mathrm{ch}^{-n / 2}\left\|z_{h}\right\|_{j, 2, T}, \quad 0 \leqq j<m .
\end{gathered}
$$

With these definitions the Galerkin approximation $U$ of problem $(\mathrm{P})$ is defined by:

$$
\begin{gathered}
\left(U_{t}, v_{h}\right)+\sum_{i=1}^{n}\left(F_{i}(\nabla U), v_{h i}\right)=\left(f, v_{h}\right), \quad v_{h}, U(., t) \in S_{h}, \quad t \in[0, T], \\
\left(F_{i j}(\nabla \psi)(\psi-U(0))_{j}, v_{h i}\right)=0, \quad v_{h} \in S_{h} .
\end{gathered}
$$

THEOREM: Let $m \geqq 3$. Under the conditions (A1)-(A5) and (S1)-(S3) there is a $h_{0}$ such that for all $0<h \leqq h_{0}$, the system $\left(\mathrm{P}^{h}\right)$ has a unique solution $U$ in $\Omega \times[0, T]$. Furthermore, the following inequality holds:

$$
\|u-U\|_{\infty} \leqq \mathrm{ch}^{m}|\ln h|^{(n / 2)+2} .
$$

REMARK: Under similar assumptions the theorem is valid for the more general quasilinear initial boundary value problem:

$$
u_{t}-\sum_{i=1}^{n} \partial_{i} F_{i}(x, t, u, \nabla u)+F(x, t, u, \nabla u)=f(x, t) .
$$

But all essential difficulties arising from the nonlinearity will be preserved in problem (P). In the case:

$$
u_{t}-\sum_{i=1}^{n} \partial_{i}\left(F_{i}(x, t, u) u_{i}\right)+F(x, t, u)=f(x, t),
$$

the method of our proof works for $m=2$ as well. For the general quasilinear equation, however, a much more complicated technique is used (see Frehse and Rannacher [8] for the elliptic case). 


\section{PROOF OF THE THEOREM}

The proof of the theorem is obtained by the method of Dobrowolski [5] combined with a deformation technique first described by Frehse [7]. The method consists in the following.

For each $r \in[0,1]$, consider the deformed problem:

$$
\left\{\begin{array}{c}
u_{t}^{r}-\sum_{i=1}^{n} \partial_{i} F_{i}\left(\nabla u^{r}\right)=r f \quad \text { in } \Omega \times[0, T], \\
u^{r}(x, 0)=r \psi \quad \text { in } \Omega, \quad u^{r}=0 \quad \text { on } \partial \Omega \times[0, T]
\end{array}\right.
$$

and the corresponding Galerkin-approximation:

$$
\begin{gathered}
\left(U_{t}^{r}, v_{h}\right)+\sum_{i=1}^{n}\left(F_{i}\left(\nabla U^{r}\right), v_{h i}\right)=\left(r f, v_{h}\right), \quad v_{h}, U^{r}(., t) \in S_{h}, \\
\left(F_{i j}(\nabla(r \psi))(r \psi-U(0))_{j}, v_{h i}\right)=0, \quad v_{h} \in S_{h} .
\end{gathered}
$$

Because of the local Lipschitz continuity of $F($.$) , it is clear that there is a unique$ solution $U^{r}$ in $\left[0, T_{h}\right]$. We intend to show that the solution exists in the whole interval $[0, T]$. In the case treated here this follows from the theory of ordinary differential equations anyhow, since the Dirichlet-form is positive definite. In the general case, i. e. $F_{i}=F_{i}(x, u, \nabla u)$, however this argument does not apply, but the existence of a global solution of the discrete problem follows as a by product of the deformation technique. Thus, illustrating how the proof proceeds in the general case, we have chosen this more complicated method to prove global solvability of the discrete problems. For each $h$, we define the set $\Xi_{h} \subset[0,1]$ by:

$$
\begin{aligned}
& \Xi_{h}=\left\{r \in[0,1]:\left(P_{h}^{r}\right) \text { has a solution } U^{r} \text { defined on }[0, T]\right. \\
& \text { and there holds }\left\|u^{r}-U^{r}\right\|_{r}<2 c_{1} h^{m}|\ln h|^{(n / 2)+2} \\
& \text { and } \left.\left\|\nabla U^{r}\right\|_{x},\left\|\nabla U_{t}^{r}\right\|_{r}<2 \gamma\right\},
\end{aligned}
$$

where $c_{1}$ is the constant appearing in lemma 1 and $\gamma$ refers to (A5). Here and in the following every constant $c$ or $c_{1}$ does not depend on $h$. We show that for $h \leqq h_{0}\left(c_{1}\right)$ the set $\Xi_{h}$ is not empty, closed and open with respect to $[0,1]$ and therefore must coincide with $[0,1]$, which proves the theorem since $u=u^{1}$ and $U=U^{1}$.

(i) $\Xi_{h}$ is not empty. For $r=0$ we have $u^{r}=0$ and $U^{r}=0$ because of (A3);

(ii) $\Xi_{h}$ is open in $[0,1]$. Let $r \in \Xi_{h}$. From (A5) and the theory of ordinary differential equations using a truncation argument we obtain the strict inequalities: 


$$
\begin{gathered}
\left\|u^{\rho}-U^{\rho}\right\|_{\infty}<2 c_{1} h^{m} \mid \ln h^{(n / 2)+2} ; \\
\left\|\nabla U^{r}\right\|_{\infty}, \quad\left\|\nabla U_{t}^{r}\right\|_{\infty}<2 \gamma
\end{gathered}
$$

if $\rho$ is in a neighbourhood of $r$;

(iii) $\Xi_{h}$ is closed. Let $r(j) \in \Xi_{h}$ and $r(j) \rightarrow r_{0}$. Immediately we obtain:

$$
\left\|u^{r_{0}}-U^{r_{0}}\right\|_{\infty} \leqq 2 c_{1} h^{m}|\ln h|^{(n / 2)+2}
$$

and

$$
\left\|\nabla U^{r_{0}}\right\|_{\infty}, \quad\left\|\nabla U_{t}^{r_{0}}\right\|_{\infty} \leqq 2 \gamma .
$$

Now we have to prove the strict inequalities for $r_{0}$. This is done by the following:

Lemma 1: Suppose that $\left\|\nabla U^{r}\right\|_{\infty},\left\|\nabla U_{t}^{r}\right\|_{\infty} \leqq 2 \gamma$. Then, under the hypotheses of the theorem, there holds $(e=u-U)$ :

$$
\begin{aligned}
& h^{4}|\ln h|^{c(n)} \int_{0}^{T}\left|e_{t t}^{r}(x, t)\right|^{2} d t \\
& +h^{2}|\ln h|^{c(n)} \int_{0}^{T}\left|\nabla e_{t}^{r}(x, t)\right|^{2} d t+\left\|e^{r}\right\|_{\infty}^{2} \\
& \leqq c|\ln h|^{c(n)}\left\{h^{-5}\left\|e^{r}\right\|_{\infty}^{4}+\left\|\nabla e^{r}\right\|_{\infty}^{2} \int_{0}^{T}\left|\nabla e_{t}^{r}\left(x_{1}, t\right)\right|^{2} d t\right\} \\
& +c_{1} h^{2 m}|\ln h|^{n+4} \mid,
\end{aligned}
$$

where $x \in \Omega$ is arbitrary and $x_{1}=x_{1}(h, r)$ is a fixed point in $\Omega$.

This lemma will be proved in the third section. From (S3) and

$$
\left\|u^{r}-U^{r}\right\|_{\infty} \leqq 2 c_{1} h^{m}|\ln h|^{(n / 2)+2}
$$

we obtain for $u_{h}^{r} \in S_{h}$ :

$$
\begin{aligned}
\left\|\nabla e^{r}\right\|_{\infty} & \leqq\left\|\nabla\left(u^{r}-u_{h}^{r}\right)\right\|_{\infty}+\left\|\nabla\left(U^{r}-u_{h}^{r}\right)\right\|_{\infty} \\
& \leqq \operatorname{ch}^{m-1}+\operatorname{ch}^{-1}\left\|U^{r}-u_{h}^{r}\right\|_{\infty} \\
& \leqq \operatorname{ch}^{m-1}+\operatorname{ch}^{-1}\left\|u^{r}-u_{h}^{r}\right\|_{\infty}+\operatorname{ch}^{-1}\left\|e^{r}\right\|_{\infty} \\
& \leqq \operatorname{ch}^{m-1}+c c_{1} h^{m-1}|\ln h|^{(n / 2)+2}
\end{aligned}
$$

and therefore:

$$
\left\|\nabla e^{r}\right\|_{\infty}^{2} \int_{0}^{T}\left|\nabla e_{t}^{r}\left(x_{1}, t\right)\right|^{2} d t \leqq c c_{1}^{2} h^{2 m-2}|\ln h|^{n+4} \int_{0}^{T}\left|\nabla e_{t}^{r}\left(x_{1}, t\right)\right|^{2} d t .
$$


Applying this to the inequality in lemma 1 and choosing $h \leqq h_{0}\left(c_{1}\right)$ we obtain:

$$
\begin{aligned}
& h^{4}|\ln h|^{c(n)} \int_{0}^{T}\left|e_{t t}^{r}(x, t)\right|^{2} d t \\
& \quad+h^{2}|\ln h|^{c(n)} \int_{0}^{T}\left|\nabla e_{t}^{r}(x, t)\right|^{2} d t+\left\|e^{r}\right\|_{\infty}^{2} \\
& \leqq \mathrm{ch}^{-5}|\ln h|^{c(n)}\left\|e^{r}\right\|_{\infty}^{4}+c_{1} h^{2 m}|\ln h|^{n+4} .
\end{aligned}
$$

In view of $\left\|e^{r}\right\|_{\infty} \leqq 2 c_{1} h^{m}|\ln h|^{(n / 2)+2}$ and $m \geqq 3$ this yields for $h \leqq h_{0}\left(c_{1}\right)$ :

$$
\left\|e^{r}\right\|_{\infty}<2 c_{1} h^{m}|\ln h|^{(n / 2)+2}
$$

and by the inverse relation:

$$
\left\|\nabla U^{r}\right\|_{\infty} \leqq\left\|\nabla e^{r}\right\|_{\infty}+\left\|\nabla u^{r}\right\|_{\infty}<2 \gamma, \quad h \leqq h_{0}\left(c_{1}\right) .
$$

It remains to prove $\left\|\nabla U_{t}^{r}\right\|_{\infty}<2 \gamma$. For this purpose let:

$$
\left|\nabla e_{t}^{r}\left(x_{0}, t_{0}\right)\right|=\left\|\nabla e_{t}^{r}\right\|_{\infty} \text {. }
$$

From the inequality:

$$
|f(t)| \leqq \varepsilon\left\|f_{t}\right\|_{L^{2}([0, T])}+c \varepsilon^{-1}\|f\|_{L^{2}([0, T])},
$$

$f \in H^{1}([0, T])$, which can be proved by integrating the relation $(d / d t) f^{2}=2 f f_{t}$, we obtain for any $\varepsilon>0$ :

$$
\left\|\nabla e_{t}^{r}\right\|_{\infty}^{2} \leqq \varepsilon \int_{0}^{T}\left|\nabla e_{t t}^{r}\left(x_{0}, t\right)\right|^{2} d t+c \varepsilon^{-1} \int_{0}^{T}\left|\nabla e_{t}^{r}\left(x_{0}, t\right)\right|^{2} d t
$$

Now (S3) yields:

$$
\int_{0}^{T}\left|\nabla e_{t t}^{r}\left(x_{0}, t\right)\right|^{2} d t \leqq \mathrm{ch}^{-2} \int_{0}^{T}\left|e_{t t}^{r}\left(x_{2}, t\right)\right|^{2} d t+\mathrm{ch}^{2 m-2},
$$

where $x_{2}$ is a fixed point in $\Omega$.

Choosing $\varepsilon=h^{2}$ in (2.2) we have:

$$
\left\|\nabla e_{t}^{r}\right\|_{\infty}^{2} \leqq \int_{0}^{T}\left|e_{t t}^{r}\left(x_{2}, t\right)\right|^{2} d t+\operatorname{ch}^{-2} \int_{0}^{T}\left|\nabla e_{t}^{r}\left(x_{0}, t\right)\right|^{2} d t+\operatorname{ch}^{2 m-2} .
$$

and with (2.1):

$$
\left\|\nabla e_{t}^{r}\right\|_{\infty}^{2} \leqq c_{1} \mathrm{ch}^{2 m-4}|\ln h|^{c(n)} .
$$

Therefore we have for $h \leqq h_{0}\left(c_{1}\right)$ :

$$
\left\|\nabla U_{t}^{r}\right\|_{\infty} \leqq\left\|\nabla e_{t}^{r}\right\|_{\infty}+\left\|\nabla u_{t}^{r}\right\|_{\infty}<2 \gamma .
$$




\section{PROOF OF LEMMA 1}

In this section we assume $\|\nabla U\|_{\infty},\left\|\nabla U_{t}\right\|_{\infty} \leqq 2 \gamma$ and suppress the parameter $r$. We begin by introducing some additional notation and technical tools. We use the weight functions:

$$
\sigma(.)=\left(|.-x|^{2}+\rho^{2}\right)^{1 / 2}, \quad \rho \geqq \mathrm{ch}
$$

and the corresponding weighted norms:

Obviously:

$$
\|\cdot\|_{(s)}=\left(\sum_{T \in \tau^{\natural}}\left\|\sigma^{s / 2} \cdot\right\|_{T}^{2}\right)^{1 / 2}, \quad s \in \mathbf{R} .
$$

$$
\sigma^{-1} \leqq \rho^{-1} \leqq h^{-1}, \quad|\nabla \sigma| \leqq 1, \quad \sigma \leqq c
$$

and for $\rho \geqq$ ch, $c$ sufficiently large, it follows from (S1)-(S3) that:

$$
\begin{gathered}
\inf _{v_{h} \in S_{h}}\left\|\nabla^{i}\left(v-v_{h}\right)\right\|_{(s)} \leqq \mathrm{ch}^{m-i} \sum_{j=1}^{m}\left\|\nabla^{j} v\right\|_{(s)}, \quad 0 \leqq i \leqq m-1, \\
\left\|\nabla^{i} v_{h}\right\|_{(s)} \leqq \mathrm{ch}^{-i}\left\|v_{h}\right\|_{(s)}, \quad 0 \leqq i \leqq m-1
\end{gathered}
$$

(see Nitsche [13]).

We use the notation $\|\cdot\|_{(s) i}, i \in \mathbf{N}$, to indicate that the norm is taken with a weight:

with:

$$
\sigma_{i}(.):=\left(|.-x|^{2}+\rho^{2}\right)^{1 / 2}+\left(\left|.-x_{i}\right|^{2}+\rho^{2}\right)^{1 / 2},
$$

$$
\sigma_{i}^{s}(.):=\left(|.-x|^{2}+\rho^{2}\right)^{s / 2}+\left(\left|\cdot-x_{i}\right|^{2}+\rho^{2}\right)^{s / 2}, \quad s \in \mathbf{R}
$$

where $x_{i}$ is a fixed point in $\Omega$.

For abbreviation, we define the functions:

$$
\begin{array}{ll}
a_{i j}^{h}(., .)=\int_{0}^{1} F_{i j}(\nabla U+(1-s) \nabla(u-U)) d s, & i, j=1, \ldots, n, \\
a_{i j}(., .)=F_{i j}(\nabla u) \in C^{1}(\Omega \times[0, T]), & i, j=1, \ldots, n
\end{array}
$$

and corresponding bilinear forms:

$$
a^{h}(v, w)=\left(a_{i j}^{h} v_{j}, w_{i}\right), \quad a(v, w)=\left(a_{i j} v_{j}, w_{i}\right)
$$

and the differential operator:

$$
(A v, w)=a(v, w) .
$$

With these definitions we have:

$$
\left(e_{t}, v_{h}\right)+a^{h}\left(e, v_{h}\right)=0, \quad v_{h} \in S_{h},
$$




$$
\begin{gathered}
\left(e_{t t}, v_{h}\right)+a^{h}\left(e_{t}, v_{h}\right)+\int_{\Omega} a_{i j t}^{h} e_{j} v_{h i} d x=0, \quad v_{h} \in S_{h}, \\
\left|a_{i j}-a_{i j}^{h}\right| \leqq c|\nabla e|, \\
\left|a_{i j t}-a_{i j t}^{h}\right| \leqq c\left\{|\nabla e|+\left|\nabla e_{t}\right|\right\}, \\
\left|a_{i j t}^{h}\right|,\left|a_{i j t}\right| \leqq c .
\end{gathered}
$$

To fix the initial value $U(., 0)$, we have defined in the first section:

$$
a\left(e, v_{h}\right)(0)=0, \quad v_{h} \in S_{/},
$$

which implies that $U(x, 0)$ is an "elliptic" projection of $u(x, 0)$. From Nitsche [13] we obtain the asymptotic error estimate:

$$
\|u(., 0)-U(., 0)\|_{L^{\infty}} \leqq \mathrm{ch}^{m}, \quad m \geqq 3 .
$$

By the above choice of the initial value $U(., 0)$ we can show in the appendix, theorem A1:

$$
\left\|u_{t}(., 0)-U_{t}(., 0)\right\|_{L^{\infty}} \leqq \mathrm{ch}^{m}, \quad m \geqq 3 .
$$

Now lemma 1 will be proved by a series of further lemmas.

LEMMA 3.1:

(i) $\int_{0}^{T}\|\nabla e\|_{(-n)}^{2} d t \leqq c \varepsilon^{-1} \int_{0}^{T}\|e\|_{(-n-2)}^{2} d t+\varepsilon h^{2} \int_{0}^{T}\left\|e_{t}\right\|_{(-n)}^{2} d t+\operatorname{ch}^{2 m-2}|\ln h|$;

(ii)

$$
\begin{aligned}
& \int_{0}^{T}\left\|\nabla e_{t}\right\|_{(-n)}^{2} d t \\
& \quad \leqq c \varepsilon^{-1} \int_{0}^{T}\left\|e_{t}\right\|_{(-n-2)}^{2} d t+\varepsilon h^{2} \int_{0}^{T}\left\|e_{t t}\right\|_{(-n)}^{2} d t \\
& \quad+c \int_{0}^{r}\|\nabla e\|_{(-n)}^{2} d t+c \int_{0}^{1}\|e\|_{(-n-2)}^{2} d t+\operatorname{ch}^{2 m-2}|\ln h| .
\end{aligned}
$$

Proof: (i) From (3.3) and the ellipticity of the form $a^{h}(.,$.$) , it follows that:$

$$
\begin{aligned}
& \frac{1}{2} \frac{d}{d t}\|e\|_{(-n)}^{2}+c\|\nabla e\|_{(-n)}^{2} \\
& \leqq\left(e_{t}, \sigma^{-n} e\right)+a^{h}\left(e, \sigma^{-n} e\right)+c \int_{\Omega}|\nabla e||e| \sigma^{-n-1} d x \\
& =\left(e_{1}, \sigma^{-n} e-\varphi_{h}\right)+a^{h}\left(e, \sigma^{-n} e-\varphi_{h}\right)+c \int_{\Omega}|\nabla e||e| \sigma^{-n-1} d x \\
& \leqq\left\|e_{t}\right\|_{(-n)}\left\|\sigma^{-n} e_{t}-\varphi_{h}\right\|_{(n)}+c\|\nabla e\|_{(-n)}\left\|\nabla\left(\dot{\sigma}^{-n} e-\varphi_{h}\right)\right\|_{(n)} \\
& +c\|\nabla e\|_{(-n)}\|e\|_{(-n-2)} \text {. }
\end{aligned}
$$


Now let $\varphi_{h}$ be the approximation of $\sigma^{-n} e$ in the sense of (S3).

Then we have:

$$
\left\|\sigma^{-n} e-\varphi_{h}\right\|_{(n)}^{2} \leqq \operatorname{ch}^{2 m} \sum_{j=0}^{m}\left\|\nabla^{j}\left(\sigma^{-n} e\right)\right\|_{(n)}^{2}
$$

From (3.1) and (3.2) we obtain for the term of order $m$ :

$$
\begin{aligned}
\left\|\nabla^{m}\left(\sigma^{-n} e\right)\right\|_{(n)}^{2} & \leqq c \sum_{j=0}^{m}\left\|\nabla^{j} e \sigma^{-n-m+j}\right\|_{(n)}^{2} \\
& \leqq c \sum_{j=0}^{m-1} h^{-2 j}\left\|e \sigma^{-n-m+j}\right\|_{(n)}^{2}+c|\ln h|
\end{aligned}
$$

Estimating $\left\|\nabla\left(\sigma^{-n} e-\varphi_{h}\right)\right\|_{(n)}$ analogously, we obtain:

$$
\begin{aligned}
& h^{-1}\left\|\sigma^{-n} e-\varphi_{h}\right\|_{(n)}+\left\|\nabla\left(\sigma^{-n} e-\varphi_{h}\right)\right\|_{(n)} \\
& \quad \leqq c\|e\|_{(-n-2)}+\operatorname{ch}^{m-1}|\ln h|^{1 / 2}
\end{aligned}
$$

By replacing (3.11) in (3.10), integrating from 0 to $T$, with accounting for $\|e\|_{(-n)}(0) \leqq \mathrm{ch}^{m}|\ln h|^{1 / 2}$, we obtain (i).

(ii) With the aid of (3.4) we get:

$$
\begin{aligned}
& \frac{1}{2} \frac{d}{d t}\left\|e_{t}\right\|_{(-n)}^{2}+c\left\|\nabla e_{t}\right\|_{(-n)}^{2} \\
& \quad \leqq\left(e_{t t}, \sigma^{-n} e_{t}\right)+a^{h}\left(e_{t}, \sigma^{-n} e_{t}\right)+c \int_{\Omega}\left|e_{t} \| \nabla e_{t}\right| \sigma^{-n-1} d x \\
& =\left(e_{t t}, \sigma^{-n} e_{t}-\varphi_{h}\right)+a^{h}\left(e_{t}, \sigma^{-n} e_{t}-\varphi_{h}\right)+\int_{\Omega} a_{i j t}^{h}\left(\sigma^{-n} e_{t}-\varphi_{h}\right)_{j} e_{i} d x \\
& \quad-\int_{\Omega} a_{i j t}^{h}\left(\sigma^{-n} e_{t}\right)_{j} e_{i} d x+c \int_{\Omega}\left|\nabla e_{t}\right| \cdot\left|e_{t}\right| \sigma^{-n-1} d x .
\end{aligned}
$$

We omit the rest of the proof, because from now on we can estimate similarly to (i).

LEMMA 3.2:

(i)

$$
\int_{0}^{T}\left\|e_{t}\right\|_{(-n)}^{2} d t \leqq \mathrm{ch}^{-2} \int_{0}^{T}\|\nabla e\|_{(-n)}^{2} d t+\operatorname{ch}^{2 m-4}|\ln h| ;
$$

(ii) $\int_{0}^{T}\left\|e_{t t}\right\|_{(-n)}^{2} d t \leqq \mathrm{ch}^{-2} \int_{0}^{T}\left\{\left\|\nabla e_{t}\right\|_{(-n)}^{2}+\|\nabla e\|_{(-n)}^{2}\right\} d t+\operatorname{ch}^{2 m-4}|\ln h|$. 
Proof: (i) From (3.3) we obtain:

$$
\begin{aligned}
\left\|e_{t}\right\|_{(-n)}^{2}=\left(e_{t}, \sigma^{-n} e_{t}\right)= & \left(e_{t}, \sigma^{-n} e_{t}-\varphi_{h}\right)+a^{h}\left(e, \sigma^{-n} e_{t}-\varphi_{h}\right)-a^{h}\left(e, \sigma^{-n} e_{t}\right) \\
\leqq & \left\|e_{t}\right\|_{(-n)}\left\|\sigma^{-n} e_{t}-\varphi_{h}\right\|_{(n)}+c\|\nabla e\|_{(-n)}\left\|\nabla\left(\sigma^{-n} e_{t}-\varphi_{h}\right)\right\|_{(n)} \\
& +\|\nabla e\|_{(-n)}\left\|\nabla e_{t}\right\|_{(-n)} .
\end{aligned}
$$

By a technique used in the proof of lemma 3.1 (i) we can show:

$$
h^{-1}\left\|\sigma^{-n} e_{t}-\varphi_{h}\right\|_{(n)}+\left\|\nabla\left(\sigma^{-n} e_{t}-\varphi_{h}\right)\right\|_{(n)} \leqq c\left\|e_{t}\right\|_{(-n-2)}+\operatorname{ch}^{m-1}|\ln h|^{1 / 2} .
$$

Then we have with the inequality:

$$
\begin{gathered}
a b \leqq \frac{1}{2} \varepsilon a^{2}+\frac{1}{2} \varepsilon^{-1} b^{2} \quad \text { and } \quad \rho \geqq \mathrm{ch}, c \text { large: } \\
\left\|e_{t}\right\|_{(-n)}^{2} \leqq \mathrm{ch}^{-2}\|\nabla e\|_{(-n)}^{2}+\varepsilon h^{2}\left\|\nabla e_{t}\right\|_{(-n)}^{2} .
\end{gathered}
$$

By the technical tool:

$$
\|\nabla v\|_{(-n)} \leqq \mathrm{ch}^{-1}\|v\|_{(-n)}+\mathrm{ch}^{m-1}|\ln h|^{1 / 2}, \quad v \in H^{m}(T), \quad T \in \tau h,
$$

which we have already used in the first section and by integrating from 0 to $T$, we have shown the lemma.

(ii) Here we use (3.4):

$$
\begin{aligned}
\left\|e_{t t}\right\|_{(-n)}^{2}= & \left(e_{t t}, \sigma^{-n} e_{t t}-\varphi_{h}\right)+a^{h}\left(e_{t}, \sigma^{-n} e_{t t}-\varphi_{h}\right) \\
& -a^{h}\left(e_{t}, \sigma^{-n} e_{t t}\right)+\int_{\Omega} a_{i j t}^{h} e_{j}\left(\sigma^{-n} e_{t t}-\varphi_{h}\right)_{i} d x \\
& -\int_{\Omega} a_{i j t}^{h} e_{j}\left(\sigma^{-n} e_{t t}\right)_{i} d x .
\end{aligned}
$$

Again we can omit the easy estimates.

Lemma 3.3:

$$
\int_{0}^{T}\|e\|_{(-n-2)}^{2} d t \leqq \mathrm{ch}^{-4}\|e\|_{\infty}^{3}+\operatorname{ch}^{2 m-4}\|e\|_{\infty}+\operatorname{ch}^{2 m-2}|\ln h| .
$$

Proof: We define the problem:

$$
\left.\begin{array}{rr}
-v_{t}-A v=\sigma^{-n-2} e & \text { in } \Omega \times[0, T], \\
v(x, t)=0 \quad \text { on } \partial \Omega \times[0, T], & v(x, T)=0 \text { in } \Omega,
\end{array}\right\}
$$


which arises from the usual parabolic equation in $\tau \in[0, T]$ by the transformation $t(\tau)=T-\tau$ so that the $a$ priori estimates stated in the appendix can be used. With the aid of (3.13), we have:

$$
\begin{aligned}
\int_{0}^{T}\|e\|_{(-n-2)}^{2} d t= & -\int_{0}^{T}\left(e, v_{t}\right) d t+\int_{0}^{T} a(e, v) d t \\
= & \int_{0}^{T}\left(e_{t}, v-v_{h}\right) d t+\int_{0}^{T} a^{h}\left(e, v-v_{h}\right) d t \\
& +\int_{0}^{T}\left(a-a^{h}\right)(e, v) d t+(e, v)(0) \\
\leqq & \int_{0}^{T}\left\|e_{t}\right\|_{(-n)}\left\|v-v_{h}\right\|_{(n)} d t+c \int_{0}^{T}\|\nabla e\|_{(-n)}\left\|\nabla\left(v-v_{h}\right)\right\|_{(n)} \\
& +\int_{0}^{T} \int_{\Omega}|\nabla e|^{2}|\nabla v| d t+\|e\|_{L^{\infty}}(0)\|v\|_{L^{1}(0)} \\
= & A+B+C+D .
\end{aligned}
$$

From the approximation property in weighted norms (3.1) and from theorem A2 in the appendix, we obtain:

$$
\begin{aligned}
A & \leqq \mathrm{ch}^{2} \int_{0}^{T} \sum_{i=0}^{2}\left\|e_{t}\right\|_{(-n)}\left\|\nabla^{i} v\right\|_{(n)} d t \\
& \leqq \varepsilon h^{2} \int_{0}^{T}\left\|e_{t}\right\|_{(-n)}^{2} d t+c \varepsilon^{-1} h^{2} \rho^{-2}|\ln h| \int_{0}^{T}\|e\|_{(-n-2)}^{2} d t
\end{aligned}
$$

and similarly

$$
\begin{aligned}
B & \leqq \mathrm{ch} \int_{0}^{T} \sum_{i=0}^{2}\|\nabla e\|_{(-n)}\left\|\nabla^{i} v\right\|_{(n)} d t \\
& \leqq \varepsilon \int_{0}^{T}\|\nabla e\|_{(-n)}^{2} d t+c \varepsilon^{-1} h^{2} \rho^{-2}|\ln h| \int_{0}^{T}\|e\|_{(-n-2)}^{2} d t .
\end{aligned}
$$

Theorems A2 (i) and (3.12) yield:

$$
\begin{aligned}
C & \leqq \nabla e\left\|_{\infty}^{2} \int_{0}^{T}\right\| \nabla v\left\|_{L^{1}} d t \leqq\right\| \nabla e\left\|_{\infty}^{2} \cdot \mathrm{ch}^{-2}\right\| e \|_{\infty} \\
& \leqq \mathrm{ch}^{-4}\|e\|_{\infty}^{3}+\operatorname{ch}^{2 m-4}\|e\|_{\infty}
\end{aligned}
$$

and theorems A2 and (3.8) yield:

$$
D \leqq \operatorname{ch}^{m} \int_{0}^{T} \int_{\Omega}\left|e \sigma^{-n-2}\right| d x d t \leqq c \varepsilon^{-1} h^{2 m-2}+\varepsilon \int_{0}^{T}\|e\|_{(-n-2)}^{2} d t .
$$


Choosing $\rho=\operatorname{ch}|\ln h|^{1 / 2}, c$ sufficiently large, and combıning the above estımates with lemmas (3.1) (i) and (3.2) (i) we obtain lemma 3.3.

LEMMA 3.4:

$$
\begin{aligned}
\int_{0}^{T}\left\|e_{t}\right\|_{(-n-2)}^{2} d t \leqq & \mathrm{ch}^{-7}\|e\|_{\infty}^{4}+\mathrm{ch}^{-1}|\ln h|^{n+5}\|e\|_{\infty}^{2} \\
& +\mathrm{ch}^{-2}\|\nabla e\|_{\infty}^{2} \int_{0}^{T}\left\|\nabla e_{t}\right\|_{(-n) 0}^{2} d t+\mathrm{ch}^{2 m-2}|\ln h|^{n+5} .
\end{aligned}
$$

Proof: Again we use the problem:

$$
\begin{gathered}
-v_{t}-A v=\sigma^{-n-2} e_{t} \quad \text { in } \Omega \times[0, T], \\
v(x, t)=0 \quad \text { on } \partial \Omega \times[0, T], \quad v(x, T)=0 \quad \text { in } \Omega,
\end{gathered}
$$

as a device to obtain:

$$
\begin{aligned}
\int_{0}^{T}\left\|e_{t}\right\|_{(-n-2)}^{2} d t= & -\int_{0}^{T}\left(e_{t}, \imath_{t}\right) d t+\int_{0}^{T} a\left(e_{t}, v\right) d t \\
= & \int_{0}^{T}\left(e_{t t}, v-v_{h}\right) d t+\int_{0}^{T} a^{h}\left(e_{t}, v-\iota_{h}\right) \\
& +\int_{0}^{T} \int_{\Omega}\left(a_{\imath \jmath t}^{h}-a_{\imath t t}\right) e_{\jmath}\left(v-v_{h}\right)_{t} d x d t \\
& +\int_{0}^{T} \int_{\Omega} a_{\imath t t} e_{\jmath}\left(v-v_{h}\right)_{\imath} d x d t+\int_{0}^{T} \int_{\Omega}\left(a_{t \jmath t}^{h}-a_{\imath \jmath t}\right) e_{\jmath} v_{\imath} d x d t \\
& -\int_{0}^{T} \int_{\Omega} a_{\imath \jmath t} e_{\jmath} v_{t} d x d t+\int_{0}^{T}\left(a-a^{h}\right)\left(v, e_{t}\right) d t+\left(v, e_{t}\right)(0) \\
= & A+B+C+D+E+F+G+H .
\end{aligned}
$$

We only estımate $C, E, F$ and $G$.

From (3.6) we conclude:

$$
\begin{aligned}
|C| & \leqq \int_{0}^{T} \int_{\Omega}\left(|\nabla e|+\left|\nabla e_{t}\right|\right)\left|\nabla e \| \nabla\left(v-v_{h}\right)\right| d x d t \\
& \leqq \operatorname{ch}\|\nabla e\|_{\infty} \int_{0}^{T}\left(\|\nabla e\|_{(-n)}+\left\|\nabla e_{t}\right\|_{(-n)}\right) \sum_{k=0}^{2}\left\|\nabla^{k} v\right\|_{(n)}
\end{aligned}
$$

and from theorem A2 and the inequality:

$$
a b \leqq \frac{1}{2} \varepsilon a^{2}+\frac{1}{2} \varepsilon^{-1} b^{2},
$$


$C \leqq c|\ln h|\|\nabla e\|_{\infty}^{4}+c\|\nabla e\|_{\infty}^{2} \int_{0}^{T}\left\|\nabla e_{t}\right\|_{(-n)}^{2} d t+\operatorname{ch}^{2} \rho^{-2}|\ln h| \int_{0}^{T}\|e\|_{(-n-2)}^{2} d t$.

Choosing $\rho=\operatorname{ch}|\ln h|^{1 / 2}, c$ sufficiently large, and applying (3.12) we see that $C$ can be estimated appropriately.

For the next term we obtain similarly:

$$
\begin{aligned}
|E| \leqq & \int_{0}^{T} \int_{\Omega}\left(|\nabla e|+\left|\nabla e_{t}\right|\right)|\nabla e \| \nabla v| d x d t \\
\leqq & \operatorname{ch}^{-2}\|\nabla e\|_{\infty}^{2} \int_{0}^{T}\left\{\|\nabla e\|_{(-n)}^{2}+\left\|\nabla e_{t}\right\|_{(-n)}^{2}\right\} d t \\
& +\operatorname{ch}^{2} \rho^{-2}|\ln h| \int_{0}^{T}\|e\|_{(-n-2)}^{2} d t .
\end{aligned}
$$

We now estimate $F$. Partial integration leads us to:

$$
|F| \leqq c \int_{0}^{T} \int_{\Omega}|e|\left|\nabla^{2} v\right| d x d t \leqq c|\ln h|^{1 / 2}\|e\|_{\infty} \int_{0}^{T}\left\|\nabla^{2} v\right\|_{(n)} d t
$$

and by theorem $\mathrm{A} 1$ :

$$
|F| \leqq \mathrm{ch}^{-2}|\ln h|\|e\|_{\infty}^{2}+\operatorname{ch}^{2} \rho^{-2}|\ln h| \int_{0}^{T}\|e\|_{(-n-2)}^{2} d t .
$$

The second term can be cancelled by altering the constant $c$ in $\rho=\operatorname{ch}|\ln h|^{1 / 2}$. Let $\left|e\left(x_{2}, t_{2}\right)\right|=\|e\|_{\infty}$. Then we get by the inequality:

$$
|f(t)| \leqq c \varepsilon^{-1}\|f\|_{L^{2}([0, T])}+\varepsilon\left\|f_{t}\right\|_{L^{2}([0, T])},
$$

$f \in H^{1}([0, T])$, and by the definition of $\sigma$ :

$$
\begin{aligned}
\|e\|_{\infty}^{2} \leqq & \varepsilon \int_{0}^{T} e_{t}\left(x_{2}, t\right)^{2} d t+c \varepsilon^{-1} \int_{0}^{T} e\left(x_{2}, t\right)^{2} d t \\
\leqq & c \varepsilon h^{-n} \rho^{n+2} \int_{0}^{T}\left\|e_{t}\right\|_{(-n-2) 1}^{2} d t \\
& +c \varepsilon^{-1} h^{-n} \rho^{n+2} \int_{0}^{T}\|e\|_{(-n-2) 0}^{2} d t+\operatorname{ch}^{2 m} .
\end{aligned}
$$

Choosing $\varepsilon=\varepsilon_{0} h^{2}|\ln h|^{(n / 2)+2}$, we arrive at:

$$
\begin{aligned}
\|e\|_{\infty}^{2} \leqq & \varepsilon_{0} h^{2}|\ln h|^{-1} \int_{0}^{T}\left\|e_{t}\right\|_{(-n-2) 1}^{2} d t \\
& +c \varepsilon_{0}^{-1} h^{2}|\ln h|^{n+3} \int_{0}^{T}\|e\|_{(-n-2) 0}^{2} d t+\mathrm{ch}^{2 m}
\end{aligned}
$$


and therefore:

$$
|F| \leqq c|\ln h|^{n+4} \int_{0}^{T}\|e\|_{(-n-2) 0}^{2} d t+\operatorname{ch}^{2 m-2}|\ln h|
$$

and by lemma 3.3:

$$
|F| \leqq \mathrm{ch}^{-4}|\ln h|^{c(n)}\|e\|_{\infty}^{3}+\mathrm{ch}^{2 m-4}|\ln h|^{c(n)}\|e\|_{\infty}+\mathrm{ch}^{2 m-2}|\ln h|^{n+5}
$$

Finally we have:

$$
|G| \leqq c \int_{0}^{T} \int_{\Omega}\left|\nabla e\left\|\nabla e_{t}\right\| \nabla v\right| d x d t
$$

Note that $|G|$ is similar to $|E|$.

Utilizing the above estimates and lemmas 3.1-3.3 we have:

$$
\begin{aligned}
\int_{0}^{T}\left\|e_{t}\right\|_{(-n-2)}^{2} d t \leqq & \operatorname{ch}^{-4}|\ln h|^{c(n)}\|e\|_{\infty}^{3}+\operatorname{ch}^{2 m-4}|\ln h|^{c(n)}\|e\|_{\infty} \\
& +\operatorname{ch}^{-2}|\ln h|^{c(n)}\|\nabla e\|_{\infty}^{4}+\operatorname{ch}^{-2}\|\nabla e\|_{\infty}^{2} \int_{0}^{T}\left\|\nabla e_{t}\right\|_{(-n) 0}^{2} d t \\
& +\operatorname{ch}^{2 m-2}|\ln h|^{n+5} .
\end{aligned}
$$

Now the technique from (3.12) and the inequality:

$$
a b \leqq \frac{1}{2} \varepsilon a^{2}+\frac{1}{2} \varepsilon^{-1} b^{2},
$$

will complete the proof.

\section{Lemma 1:}

$$
\begin{aligned}
& h^{4}|\ln h|^{c(n)} \int_{0}^{T}\left|e_{t t}(x, t)\right|^{2} d t+\operatorname{ch}^{2}|\ln h|^{c(n)} \int_{0}^{T}\left|\nabla e_{t}(x, t)\right|^{2} d t+\|e\|_{\infty}^{2} \\
& \leqq c|\ln h|^{c(n)}\left\{h^{-5}\|e\|_{\infty}^{4}+\|\nabla e\|_{\infty}^{2} \int_{0}^{T}\left|\nabla e_{t}\left(x_{1}, t\right)\right|^{2} d t\right\}+c_{1} h^{2 m}|\ln h|^{n+4},
\end{aligned}
$$

where $x_{1}=x_{1}(h)$ is a fixed point in $\Omega$.

Proof: From the definition of weighted norms we conclude:

$$
\begin{aligned}
& \int_{0}^{T}\left|e_{t t}(x, t)\right|^{2} d t+\int_{0}^{T}\left|\nabla e_{t}(x, t)\right|^{2} d t \\
& \quad \leqq c|\ln h|^{c(n)} \int_{0}^{T}\left\{\left\|e_{t t}\right\|_{(-n)}^{2}+\left\|\nabla e_{t}\right\|_{(-n)}^{2}\right\} d t+\mathrm{ch}^{2 m-2}|\ln h|^{c(n)}
\end{aligned}
$$


Now lemmas 3.1 (ii), 3.2 (ii) and 3.4 yield the statement for the first two terms. From the inequality:

$$
|f(t)| \leqq \varepsilon\left\|f_{t}\right\|_{L^{2}([0, T))}+c \varepsilon^{-1}\|f\|_{L^{2}([0, T])}, \quad f \in H^{1}([0, T]),
$$

we conclude for $\left|e\left(x_{0}, t_{0}\right)\right|=\|e\|_{\infty}$ :

$$
\|e\|_{\infty}^{2} \leqq \varepsilon \int_{0}^{T} e_{t}\left(x_{0}, t\right)^{2} d t+c \varepsilon^{-1} \int_{0}^{T} e\left(x_{0}, t\right)^{2} d t+\operatorname{ch}^{2 m}
$$

and from $\varepsilon=|\ln h|^{(-n / 2)-2}$ and the definition of $\sigma$, it follows that

$$
\|e\|_{\infty}^{2} \leqq \mathrm{ch}^{2}|\ln h|^{-1} \int_{0}^{T}\left\|e_{t}\right\|_{(-n-2) 0}^{2} d t+\mathrm{ch}^{2}|\ln h|^{n+3} \int_{0}^{T}\|e\|_{(-n-2) 0}^{2} d t+\mathrm{ch}^{2 m} .
$$

Now by the lemmas 3.1-3.4:

$$
\begin{aligned}
\|e\|_{\infty}^{2} \leqq & \operatorname{ch}^{-5}|\ln h|^{c(n)}\|e\|_{\infty}^{4}+\operatorname{ch}|\ln h|^{c(n)}\|e\|_{\infty}^{2} \\
& +c|\ln h|^{c(n)}\|e\|_{\infty} \int_{0}^{T}\left\|\nabla e_{t}\right\|_{(-n) 0} d t \\
& +\operatorname{ch}^{-2}|\ln h|^{n+3}\|e\|_{\infty}^{3} \\
& +\operatorname{ch}^{2 m-2}|\ln h|^{c(n)}\|e\|_{\infty}+\mathrm{ch}^{2 m}|\ln h|^{n+4} .
\end{aligned}
$$

Finally, we obtain from the theorem of Fubini:

$$
\int_{0}^{T}\left\|\nabla e_{t}\right\|_{(-n) 0}^{2} d t \leqq c|\ln h| \int_{0}^{T}\left|\nabla e_{t}\left(x_{1}, t\right)\right| d t
$$

\section{APPENDIX}

THEOREM A1: For the solution u of problem $(\mathrm{P})$ and the corresponding Galerkinapproximation $U$ of $\left(\mathrm{P}_{h}\right)$, we have the asymptotic error estimate:

$$
\left\|u_{t}(., 0)-U_{t}(., 0)\right\|_{L^{\infty}} \leqq \mathrm{ch}^{m}, \quad m \geqq 3 .
$$

Proof: In view of:

$$
a\left(u-U, v_{h}\right)(0)=0, \quad v_{h} \in S_{h},
$$

we have with $e=u-U$ for $t=0$ :

$$
\left(e_{t}, v_{h}\right)+\left(a-a^{h}\right)\left(e, v_{h}\right)=0, \quad v_{h} \in S_{h} .
$$

vol. $12, \mathrm{n}^{\circ} 3,1978$ 
Using the weight function $\sigma$ and $q=n+\delta, \delta \in(0,1)$, we obtain:

$$
\begin{aligned}
\left(e_{t}, \sigma^{-q} e_{t}\right)= & \left(e_{t}, \sigma^{-q} e_{t}-\varphi_{h}\right)+\left(a-a^{h}\right)\left(e, \sigma^{-q} e-\varphi_{h}\right) \\
& -\left(a-a_{h}\right)\left(e, \sigma^{-q} e\right), \quad t=0 .
\end{aligned}
$$

Choosing $\varphi_{h}=$ approximation of $\sigma^{-q} e$ we can estimate:

$$
\begin{aligned}
\left\|e_{t}\right\|_{(-q)}^{2} \leqq & \operatorname{ch}\left\|e_{t}\right\|_{(-q)} \sum_{i=0}^{1}\left\|\nabla^{i}\left(\sigma^{-q} e_{t}\right)\right\|_{(q)} \\
& +c\|\nabla e\|_{\infty}\|\nabla e\|_{(-q)}\left\|\nabla\left(\sigma^{-q} e\right)\right\|_{(q)}, \quad t=0 .
\end{aligned}
$$

Now we choose $\rho=$ ch with $c$ large and get:

$$
\left\|e_{t}\right\|_{(-q)}^{2} \leqq \mathrm{ch}^{2 m-\delta} .
$$

If

$$
\left|e_{t}\left(x_{0}, 0\right)\right|=\left\|e_{t}(., 0)\right\|_{\infty}
$$

and

$$
\sigma(.)=\left(\left|\cdot-x_{0}\right|^{2}+\rho^{2}\right)^{1 / 2}
$$

we obtain from (A1):

$$
\left\|e_{t}(., 0)\right\|_{L^{\infty}} \leqq \mathrm{ch}^{m}
$$

TheOREM A2: Let $-A v:=-\left(a_{i j}(x, t) v_{j}\right)_{i}$ be a sufficiently regular elliptic differential operator and let $v(x, t)$ be the solution of the problem:

$$
\left\{\begin{array}{c}
v_{t}-\mathrm{A} v=f(x, t) \quad \text { in } \Omega \times(0, T], \\
v(x, t)=0 \quad \text { on } \partial \Omega \times[0, T], \quad v(x, 0)=0 \quad \text { in } \Omega,
\end{array}\right.
$$

where $f \in L^{2}(\Omega \times[0, T])$. Then:

$$
\int_{0}^{T} \sum_{i=0}^{2}\left\|\nabla^{i} v\right\|_{(n)}^{2} d t \leqq c \rho^{-2}|\ln h| \int_{0}^{T}\|f\|_{(n+2)}^{2} d t .
$$

Proof: Let us start with the case $n \geqq 3$. We have:

$$
\left(\sigma^{n / 2} v\right)_{t}-A\left(\sigma^{n / 2} v\right)=\sigma^{n / 2} v_{t}-\sigma^{n / 2} A v-v A \sigma^{n / 2}-2 a_{i j}\left(\sigma^{n / 2}\right)_{j} v_{i}
$$

Because $\left(\sigma^{n / 2} v\right)(x, 0)=0$ and the boundary condition is fulfilled the standard a priori estimate yields:

$$
\int_{0}^{T}\left\|\nabla^{2}\left(\sigma^{n / 2} v\right)\right\|^{2} d t \leqq c \int_{0}^{T}\left\{\left\|v_{t}-A v\right\|_{(n)}^{2}+\|\nabla v\|_{(n-2)}^{2}+\|v\|_{(n-4)}^{2}\right\} d t
$$


and therefore:

$$
\int_{0}^{T}\left\|\nabla^{2} v\right\|_{(n)}^{2} d t \leqq c \int_{0}^{T}\left\{\|f\|_{(n)}^{2}+\|\nabla v\|_{(n-2)}^{2}+\|v\|_{(n-4)}^{2}\right\} d t .
$$

Further we conclude by partial integration:

$$
\begin{gathered}
\|\nabla v\|_{(n-2)}^{2} \leqq \int_{\Omega}\left\{\left|\nabla^{2} v\left\|v\left|\sigma^{n-2}+\right| \nabla v\right\| v \| \nabla\left(\sigma^{n-2}\right)\right|\right\} d x \\
\leqq c\|v\|_{(n-4)}\left\{\left\|\nabla^{2} v\right\|_{(n)}+\|\nabla v\|_{(n-2)}\right\}
\end{gathered}
$$

and by the inequality:

$$
\begin{gathered}
a b \leqq \frac{1}{2} \varepsilon a^{2}+\frac{1}{2} \varepsilon^{-1} b^{2}, \\
\|\nabla v\|_{(n-2)} \leqq \varepsilon\left\|\nabla^{2} v\right\|_{(n)}+c \varepsilon^{-1}\|v\|_{(n-4)} .
\end{gathered}
$$

For abbreviation, let $\Omega_{T}:=\Omega \times[0, T]$. Denoting by $\Gamma$ the Green's function of $(d / d t)-A$ over $\Omega_{T}$ we obtain:

$$
\int_{0}^{T}\|v\|_{(n-4)}^{2}=\int_{\Omega_{T}} \sigma^{n-4}\left|\int_{\Omega_{t}}\left(\frac{d}{d t}-A\right) v \Gamma d y d \tau\right|^{2} d x d t
$$

and by Hölder's inequality and an interchange of the order of integration:

$$
\int_{0}^{T}\|v\|_{(n-4)}^{2} \leqq \int_{\Omega_{T}} \sigma^{n+2} f^{2}\left\{\int_{\Omega_{T}} \sigma^{n-4}|\Gamma|\left(\int_{\Omega_{T}} \sigma^{-n-2}|\Gamma| d x^{\prime} d t^{\prime}\right) d y d \tau\right\} d x d t
$$

It is well known that the Green's function $\Gamma$ can be estimated by the Green's function of $d / d t-c \Delta, c>0$, over $\mathbf{R}^{n} \times[0, T]$ (see [9]). Then the solution $w$ of the initial value problem:

$$
\begin{gathered}
w_{t}-c \Delta w=\sigma^{-\mu}(x), \\
w(x, 0)=0,
\end{gathered}
$$

yields an estimate of $\int_{\Omega_{T}} \sigma^{-\mu}(x)|\Gamma| d x d t$. Denoting by $w_{1}$ the solution of the corresponding elliptic problem:

$$
-c \Delta w_{1}=\sigma^{-\mu}(x)
$$

it is clear that:

$$
w(x, t) \leqq w_{1}(x)
$$

From:

$$
-\Delta \sigma^{2-n}=n(n-2) \rho^{2} \sigma^{-n-2}
$$

vol. $12, \mathrm{n}^{\circ} 3,1978$ 
and

$$
-\Delta\left(\ln \sigma^{-2}\right)=\left(2(n-2)\left|y-x_{0}\right|^{2}+2 n \rho^{2}\right) \sigma^{-4},
$$

we easily obtain:

$$
\int_{\Omega_{\top}} \sigma^{-n-2}|\Gamma| d x^{\prime} d t^{\prime} \leqq c \rho^{-2} \sigma^{2-n}
$$

and

and therefore:

$$
\int_{\Omega_{T}} \sigma^{-2}|\Gamma| d y d \tau \leqq c|\ln \rho|
$$

$$
\int_{0}^{T}\|v\|_{(n-4)}^{2} d t \leqq c \rho^{-2}|\ln h| \int_{0}^{T}\|f\|_{(n+2)}^{2} d t
$$

Together with (A3), (A4) this completes the proof for $n \geqq 3$. Denoting by $y^{k}=x^{k}-x_{0}^{k}, j=1,2$, the components of the vector $x-x_{0}, x \in \Omega$, we get for $n=2$ :

$$
\left\|\nabla^{2} v\right\|_{(2)}^{2}=\sum_{j=1}^{2}\left\|y^{k} \nabla^{2} v\right\|^{2}+\rho^{2}\left\|\nabla^{2} v\right\|^{2}
$$

and by the standard a priori estimate, we have:

$$
\int_{0}^{T} \rho^{2}\left\|\nabla^{2} v\right\|^{2} d t \leqq c \int_{0}^{T} \rho^{2}\|f\|^{2} d t \leqq c \rho^{-2} \int_{0}^{T}\|f\|_{(4)}^{2} d t .
$$

Now we observe that $y^{k} v, k=1,2$, is the solution of the problem:

$$
y^{k} v_{t}-A\left(y^{k} v\right)=y^{k} v_{t}-y^{k} A v-v A y^{k}-2 a_{i j} y_{j}^{k} v_{i}, \quad k=1,2
$$

and again from the standard a priori estimate we conclude:

$$
\int_{0}^{T}\left\|\nabla^{2}\left(y^{k} v\right)\right\|^{2} d t \leqq \int_{0}^{T}\left\|y^{k} f\right\|^{2} d t+c \int_{0}^{T}\|v\|_{1,2}^{2} d t, \quad k=1,2 .
$$

Hence:

$$
\begin{aligned}
\int_{0}^{T}\left\|y^{k} \nabla^{2} v\right\|^{2} d t & \leqq c \int_{0}^{T}\left\{\left\|\nabla^{2}\left(y^{k} v\right)\right\|^{2}+\|\nabla v\|^{2}\right\} d t \\
& \leqq c \int_{0}^{T}\left\{\|f\|_{(2)}^{2}+\|v\|_{1,2}^{2}\right\} d t, \quad k=1,2
\end{aligned}
$$

and thus by Poincaré's inequality and $\sigma^{-1} \leqq \rho^{-1}$ :

$$
\int_{0}^{T}\left\|\nabla^{2} v\right\|_{(2)}^{2} d t \leqq c \int_{0}^{T}\left\{\|f\|_{(2)}^{2}+\|\nabla v\|^{2}\right\} d t .
$$


By partial integration, it follows that:

$$
\begin{aligned}
\int_{0}^{T}\|\nabla v\|^{2} d t & \leqq c \int_{0}^{T} a(v, v) d t \leqq-c \int_{0}^{T} \int_{\Omega}\left(v_{t}-A v\right) v d x d t \\
& \leqq c \rho^{-2}|\ln \rho| \int_{0}^{T}\|f\|_{(4)}^{2} d t+c \rho^{2}|\ln \rho|^{-1} \int_{0}^{T}\|v\|_{(-4)}^{2} d t .
\end{aligned}
$$

Denoting by $\Gamma$ the Green's function of $(d / d t)-A$ over $\Omega \times[0, T]$ we obtain:

$$
\begin{aligned}
\int_{0}^{T}\|v\|_{(-4)}^{2} d t & =\int_{\Omega_{T}} \sigma^{-4}\left|\int_{\Omega_{T}}\left(\frac{d}{d t}-A\right) v \Gamma d y d \tau\right|^{2} d x d t \\
& \left.\leqq \int_{\Omega_{T}} \sigma^{4} f\left\{\int_{\Omega_{T}} \sigma^{-4}|\Gamma| \int_{\Omega_{T}} \sigma^{-4}|\Gamma| d x^{\prime} d t^{\prime}\right) d y d \tau\right\} d x d t .
\end{aligned}
$$

Analogously to the case $n \geqq 3$, it follows that:

$$
\int_{0}^{T}\|v\|_{(-4)}^{2} d t \leqq c \rho^{-4}(1+|\ln \rho|)^{2} \int_{0}^{T}\|f\|_{(4)}^{2} d t
$$

and thus:

$$
\int_{0}^{T}\|\nabla v\|^{2} d t \leqq c \rho^{-2}(1+|\ln \rho|) \int_{0}^{T}\|f\|_{(4)}^{2} d t .
$$

Now the theorem is proved by (A5) and $\rho(h) \geqq$ ch.

THEOREM A3: If $v$ is the solution of problem (A1), then:

(i)

$$
\begin{gathered}
\int_{0}^{T}\|\nabla v\|_{L^{1}} d t \leqq c \int_{0}^{T}\|f\|_{L^{1}} d t ; \\
\|v\|_{L^{1}}(t) \leqq c \int_{0}^{T}\|f\|_{L^{1}} d t .
\end{gathered}
$$

Proof: (i) Denoting by $\Gamma$ the Green's function of problem (A1) we have:

$$
\int_{0}^{T} \int_{\Omega}|\nabla v| d x d t \leqq \int_{0}^{T} \int_{\Omega} \int_{0}^{T} \int_{\Omega}\left|\nabla_{x} \Gamma(x-y, t-\tau) f(y, \tau)\right| d y d \tau d x d t
$$

and by the theorem of Fubini:

$$
\leqq \int_{0}^{T} \int_{\Omega}|f(y, \tau)|\left\{\int_{0}^{T} \int_{\Omega}\left|\nabla_{x} \Gamma(x-y, t-\tau)\right| d x d t\right\} d y d \tau .
$$

It is well known that:

$$
\left|\nabla_{x} \Gamma(x, t)\right| \leqq c t^{-(n+1) / 2} \exp \left(-c \frac{|x|^{2}}{t}\right)
$$

and the right hand side is integrable over $\mathbf{R}^{n} \times[0, T]$ (see [9]).

(ii) The proof is similar to (i). 


\section{REFERENCES}

1. J H. Bramble and S. R Hilbert, Bounds on a Class of Linear Functionals with Applications to Hermite Interpolation, Numer. Math., Vol 16, 1971, pp. 362-369

2. J H. Bramble, A. W. Schatz, V. Thomee and L. Wahlbin, Some Convergence Estimates for Semidiscrete Galerkin Type Approximations for Parabolic Equations, S.I.A M. J. Numer Anal, Vol. 14, 1977, pp 218-241.

3 P. G. Ciarlet and P A. RaviaRt, General Lagrange and Hermite Interpolation in $\mathbf{R}^{n}$ with Applications to the Finite Element Method, Arch Rat. Mech Anal., Vol. 46, 1972, pp 177-199

4. P. G. Ciarlet and P A Raviart, The Combined Effect of Curved Boundarles and Numerical Integration in the Isoparametric Finite Element Method in The Mathematical Foundation of the Finite Element Method with Applications to Partial Differential Equatıons, A. K. Aziz, Ed., Academic Press, New York, 1972

$5 \mathrm{M}$ Doвrowolski, $L^{\infty}$-Fehlerabschatzungen in der Methode der finten Elemente bet quastlinearen parabolischen Differentıal gleıchungen zwetter Ordnung, Diplomarbett No 13569, Bonn, 1976.

6. J. Douglas Jr. and T. Dupont, Galerkın Methods for Parabolic Equations, S I.A M., J. Numer. Anal., Vol. 7, 1970, pp 575-626

7. J Frehse, Optimale gleichmaßige Konvergenz der Methode der finiten Elemente be quasilinearen n-dimensionalen Rand wertproblemen, Zeitschrift Angew. Math $\mathrm{u}$. Mech., Tagungsband G.A M.M., 1976 (to appear).

8. J. Frehse and R. RanNaCher, Asymptotoc $L^{\infty}$-Error Estimates for Linear Finite Element Approximations of Quasilinear Boundary Value Problems, S.I.A.M J Numer. Anal. (to appear).

9 J. Frehse and R Rannacher, Optimal Uniform Convergence for the Finite Element Approximation of a Quasilinear Elliptic Boundary Value Problem, Preprint.

10. A. Friedman, Partial Differential Equations of Parabolic Type, Prentice-Hall, Englewood Cliffs, N. I., 1964.

11. O. A. Ladyzenskaja, V A. Solonnikov and N. N. URal'Ceva, Linear and Quasilinear Equations of Parabolic Type, American Mathematical Society, Providence, Rhode Island, 1968

12. J. L. Lions and E Magenes, Problèmes aux limites non homogènes et applications, Vol. I, II. Dunod, Parıs, 1968, 1970.

$13 \mathrm{~J}$ Nitsche, $L^{\infty}$-Convergence of Finite Element Approximation, Preprint; 2. Conference on Finte Elements, Rennes, 1975

14. R RANNACher, Some Asymptotic Error Estımates for Finite Element Approximation of Minimal Surfaces, Preprint.

15 V Thоме́E and L. WAHLbin, On Galerkın Methods in Semilinear Parabolıc Problems, S.I.A.M. J. Numer. Anal., Vol 12, 1975, pp. 378-389.

16. M. F. WheEler, A prior $L^{2}$-Error Estımates for Galerkin Approxımatıon to Parabolic Partial Differential Equatıons, S I.A M. J. Numer. Anal., Vol. 10, 1973, pp. 723-758.

17 M. Zlàmal, Curved Elements in the Finite Element Methods I S.I.A M., J Numer Anal., Vol. 10, 1973, pp. 229-249; II. S.I A.M , J. Numer Anal., Vol. 11, 1974, pp. $347-362$ 